\title{
Semantic and Syntactic Ambiguities in the Acquisition of Chinese Modal Verbs: Based on Intralingual and Interlingual Differences
}

\author{
Lai Peng \\ School of Foreign Languages, Sun Yat-sen University, China
}

Received May 11, 2020; Revised June 21, 2020; Accepted July 20, 2020

Copyright $\bigcirc 2020$ by authors, all rights reserved. Authors agree that this article remains permanently open access under the terms of the Creative Commons Attribution License 4.0 International License

\begin{abstract}
Given the complexities which lie in the Chinese modal system, many Chinese teachers may feel that it is a daunting task to teach Chinese modal verbs to learners from other countries. Taking Chinese modal verbs “会” (hui) and “能” (neng) as the focus of the study, this paper analyzes and exemplifies the ambiguities in the acquisition of Chinese modal verbs from both semantic perspective and syntactic perspective in the aim of shedding light on the polysemous and indeterminate features of Chinese modal verbs and increasing learners' tolerance of ambiguity caused by polysemy and indeterminacy. Semantic ambiguity is illustrated by the use of semantic components and fuzzy set figures. Syntactic ambiguity is revealed through exemplified inconsistencies in grammatical distinction of root modality and epistemic modality. Mixed ambiguities of the above two are shown through transfer errors made by learners of Chinese. Analysis of the three types of ambiguity is based on both intralingual differences among Chinese modal verbs and interlingual differences between Chinese and English modal systems. It is revealed that ambiguity results from one-to-more correspondence in form-meaning mapping within the Chinese modal system and one-to-more correspondence between the English and Chinese modal verbs. As tolerance of ambiguity is needed for successful acquisition, it is hoped that the ambiguities identified in this paper can help Chinese teachers find a way to increase the learners' tolerance of ambiguity and can provide a key for researchers of Chinese to unlock more inherent features of modal verbs.
\end{abstract}

Keywords Semantic Ambiguity, Syntactic Ambiguity, Tolerance of Ambiguity, Modal Acquisition, Chinese Modal Verbs

\section{Modal Verbs and Second Language Acquisition (SLA)}

Contemporary language teaching has made communicative competence its target. In terms of communication, one of the major functions of language is to express emotions, opinions and attitudes, for this purpose speakers need to resort to the use of modal verbs (i.e. "modal auxiliaries", or "modals"). Therefore, modal acquisition is crucial for the effective use of the target language in social communication, and it should be regarded as an important part in language teaching.

However, in the process of language teaching, it is found that the acquisition of modal verbs is a great difficulty for language learners. As Palmer (1990) pointed out, "there is no area of English grammar that is both more important and more difficult than the system of the modals" [1]. Celce-Murcia \& Larsen-Freeman (1999) also pointed out, "modal auxiliaries are one of the most difficult structures that you as an ESL/EFL teacher will have to deal with" [2]. The Chinese modal system is even more difficult to learn or teach than the English one. It is primarily because the form-meaning mapping of modal verbs is not one-to-one correspondence, and this is especially true in Chinese. The same modal form/verb in Chinese may have more than one meaning, while the same meaning may be expressed by more than one modal form/verb. The choice of appropriate Chinese modal verbs is heavily dependent on their semantic connotations and syntactic contexts. Intralingual complexity within the Chinese modal system makes it hard for learners of Chinese to tell apart different Chinese modals and may cause them to feel greatly confused and considerably anxious in the process of modal acquisition. Interlingual difference between Chinese and English modal systems is another source for confusion and anxiety. The degree of anxiety depends on learners' tolerance of 
ambiguity, which will be illustrated in the next section. Ambiguities in modal use may inhibit successful acquisition of Chinese modals, therefore, this paper aims at reducing anxiety in learning and increasing the learner's tolerance of ambiguity by making clear the different types of ambiguity in modal acquisition. For better exemplification, this paper will only focus on the analysis of “会” (hui) and “能” (neng) in terms of modal ambiguity from both semantic perspective and syntactic perspective.

\section{Tolerance of Ambiguity as a Cognitive Style in SLA}

Tolerance of Ambiguity (TOA) is a cognitive style in learning. As a learning style, tolerance of ambiguity refers to the degree of willingness for the learners to cognitively tolerate and accept a rule system that is different from his original knowledge system [3]. In terms of language learning, it refers to the degree of willingness for learners to tolerate ambiguity caused by linguistic differences when they tackle items in the new language. In this sense, "ambiguity" in the term "TOA" does not necessarily mean what we normally mean by "ambiguity". It means uncertainty commonly felt by second language learners due to intralingual complexity within the target language (new system) or interlingual differences between the target language and their mother tongue (original system). According to Ely (2002), uncertainty is a key word to define ambiguity, relating to ambiguous items in the second language which the learners are uncertain about when perceiving or using them [4]. Ambiguity caused by uncertainty about the new language brings about psychological anxiety or even a feeling of threat and discomfort to the learners, which may greatly inhibit the acquisition of the new language. It is in this sense that Brown (1987) pointed out that second language learning necessitates tolerance of ambiguity, which, as a psychological construct, is an important factor to consider in foreign language teaching [3].

Language learners are faced with enormous linguistic uncertainty, and this is especially true in the productive aspects such as speaking and writing. "The uncertainty may actually increase as the student becomes a more sophisticated L2 user and begins to wonder about situational appropriateness and lexical nuance" [4]. Psychological uncertainty is caused by the lack of determinacy within the new language or differences between the two languages, and the degree to tolerate uncertainty or indeterminacy shows the degree to tolerate ambiguity.

According to Brown (1987), appropriate degree of willingness to tolerate ambiguity facilitates language learning [3]. Learners with appropriate tolerance of ambiguity are willing to accept and internalize a new system and are less disturbed by ambiguity or uncertainty both cognitively and emotionally. They are less concerned about precision in using the new language and less afraid of making syntactic errors. However, learners with low tolerance of ambiguity are reluctant to accept a new system and tolerate uncertainty, as they are more concerned about precision in the use of language and more afraid of making mistakes. Learners who are intolerant of ambiguity may perceive linguistic items marked by vagueness, inconsistencies, multiple meanings, uncertain or unstructured situations as sources of psychological anxiety [4]. Low tolerance of ambiguity in second language learning may thus lead to failure in language acquisition. Therefore, it is necessary for language teachers to identify the sources of ambiguity in order to reduce anxiety in learning and increase success in acquisition.

According to the study of Chinese modal verbs by the author of this paper, there are enormous ambiguities in the process of learning and using modals, so it is thought that a relatively high degree of TOA is needed for success in modal acquisition.

Although TOA has been regarded as one of the important factors which may influence the success of SLA, there has not been an adequate research on it, and no research seems to have been done in terms of the relationship between the degree of TOA and the success rate of modal acquisition. With this as the next target, the present paper will primarily aim to identify and exemplify the ambiguities of Chinese modal verbs from semantic and syntactic perspectives, based on intralingual analysis and interlingual comparison.

\section{Semantic Ambiguity Caused by Polysemy and Indeterminacy of Modal Verbs}

\subsection{The Semantic Components of Modal Verbs}

To better interpret the meaning of Chinese modals and more clearly show the cognitive differences between them, Lai (2016) identified six semantic components which can be selectively used to describe the meaning of modal verbs [5]. They are subjectivity, objectivity, internal force, external force, possibility, and necessity. Each Chinese modal verb has more than one meaning. Each meaning can be described by the combination of some of the above semantic components. Different arrays of the semantic components when they are used to describe modal meaning may reveal the cognitive differences between different meanings of the same modal verb or between similar meanings of different modal verbs. Among the six semantic components, "possibility"/"necessity" is the common semantic core of all the different modal verbs. In other words, no matter it is expressing root modality or epistemic modality, all the modal verbs basically mean either possibility or necessity [6]. According to Palmer 
(2001), event modality (i.e. root modality) expresses possibility or necessity of an action caused by the speaker's ability, volition, obligation and permission, etc., while propositional modality (which includes epistemic modality) expresses possibility or necessity based on the speaker's judgment or inference [6]. According to the interpretation in [5], ability and volition belong to internal force within the subject of the modal verb, while obligation and permission can be regarded as a kind of external force toward the subject of the modal verb, and epistemic possibility or necessity is evaluated based on subjective or objective inference/judgment. It is in this sense that the six semantic components can be selectively used to describe modal meaning, which will be illustrated by examples in the following sections.

\subsection{Semantic Ambiguity Caused by the Polysemous Feature of Modal Verbs}

Each Chinese modal verb carries multiple meanings, as it may mean differently in different contexts. Therefore, form-meaning mapping is not one-to-one correspondence. One modal form/verb may be mapped with more than one meaning, and different modal forms may have a similar meaning. To learners, the multiple meanings of each modal verb are hard to take hold of, while similar meanings of different modal verbs are hard to tell apart. Take Chinese modal verbs “会”(hui) and “能”(neng) as examples. “会”(hui) has 3 meanings, and “能” (neng) has 5 meanings, expressing both root modality and epistemic modality. As revealed in Figure 1, being different modal forms (verbs), “会”(hui) and “能”(neng) can sometimes express the same meaning. But when it occurs, they must be used in different syntactic contexts. When they are used in the same syntactic contexts, they must mean differently. In this case, both semantic nuances between them and situational appropriateness for using them are factors to consider in actual use. At this point, as Ely (2002) indicated, the learners are faced with even greater linguistic uncertainty [4], which, brought about by complicated form-meaning mapping, may cause tremendous ambiguity for foreign learners of Chinese in terms of understanding and using modal verbs. Learners may perceive the polysemous feature of modal verbs as the source of complexity and ambiguity. Ambiguity caused by polysemy often results in anxiety in modal acquisition and failure in using the right modal verb in the right context.

As shown in Figure 1, the same modal form is mapped with more than one meaning: "hui" is mapped with 3 meanings and "neng" is mapped with 5 meanings. In this paper, "hui", when expressing ability, will be indicated as "hui $i_{v}$ " (because "hui" in this sense can be used as a real verb in Chinese, not only as a modal verb [5] ), "hui" expressing volition will be indicated as "hui $i_{1}$ ", and "hui" expressing prediction/inference will be indicated as "hui 2 ", while "neng" when expressing ability will be indicated as "neng,", "neng" expressing condition will be indicated as "neng,", "neng" expressing permission will be indicated as "neng,", "neng" expressing prediction/inference will be indicated as "neng", and "neng" expressing volition will be indicated as "neng, 5 " [5].

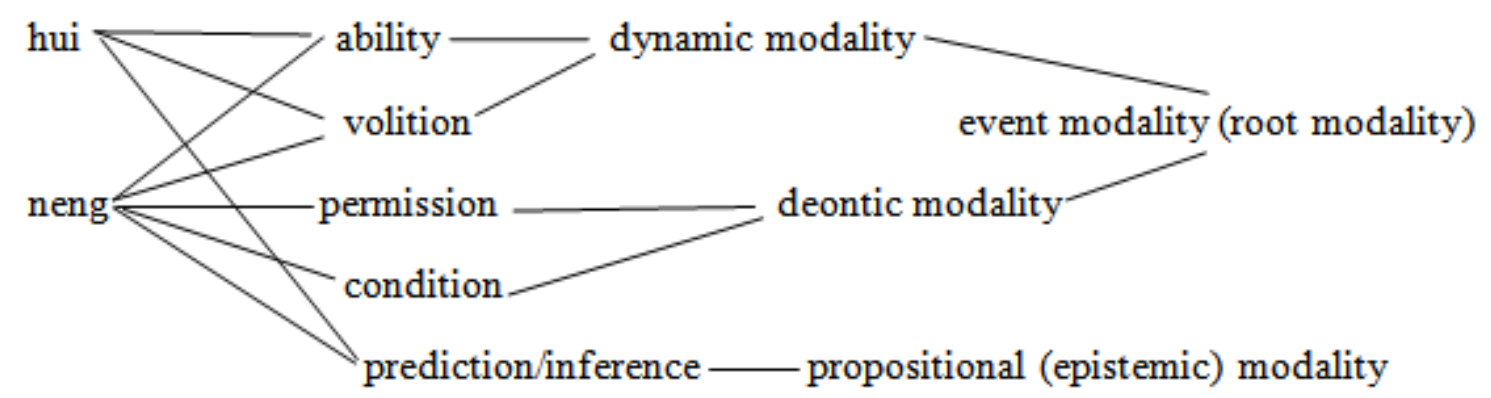

Figure 1. Form-meaning mapping of modal verb "hui" and "neng" 
In relation to the semantic components, both ability and volition are a kind of internal force within the subject of the modal verb causing the possibility or necessity of an action. Although both hui $\mathrm{i}_{\mathrm{v}}$ and neng ${ }_{1}$ express ability, hui $\mathrm{v}_{\mathrm{v}}$ means ability enabled by complete internal force within the subject of the modal verb, while neng ${ }_{1}$ may mean ability enabled not only by internal force but also by external force. For example, “他一岁就会( hui $_{\mathrm{v}}$ )走路了, 三岁的时候腿 受了伤不能( bu neng $)_{1}$ )走了, 住院治疗一个月后又能 $\left(\right.$ neng $\left._{1}\right)$ 走了(He could walk when he was only one year old, but he couldn't walk when his leg got hurt at the age of three. Then he could walk again after being hospitalized and cured for a month)". In this example, the Chinese version of the sentence first used “会”, $\left(\right.$ hui $_{v}$ ) and then “能”, (neng $)_{1}$ to mean ability (to walk), which shows semantic differentiation between "hui $\mathrm{v}_{\mathrm{v}}$ "(ability enabled by internal force) and "neng," (ability disabled or enabled by external force such as injury and hospitalization). It could be imagined that learners from other countries may have problems in putting the English version of this sentence into right Chinese wording, as they are faced with the choice of two Chinese modals ("hui ${ }_{\mathrm{v}}$ " and "neng,"). Not knowing the cognitive differences between them in terms of internal force or external force, learners of Chinese may often confuse them in actual use. Thus, hui ${ }_{v}$ and neng $g_{1}$ form a confusable pair of modals. The confusion between them may be perceived by learners as ambiguity and may result in anxiety in learning.

Although both hui $i_{1}$ and neng ${ }_{5}$ express volition, hui involves only the subjective internal force within the subject of the modal verb in the sentence, while neng ${ }_{5}$ may involve both subjective internal force within the subject of the modal verb in the sentence and subjective external force from the speaker of that sentence. In this sense, hui ${ }_{1}$ and neng ${ }_{5}$ form another confusable pair of modals.

The above two pairs of modal verbs express root modality. In terms of epistemic modality, hui ${ }_{2}$ and neng become a pair of confusable modals. Both of them show inference made by the speaker, but the difference between them is that hui ${ }_{2}$ expresses a kind of epistemic necessity based on subjective inference/judgment made by the

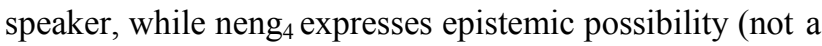
necessity) inferred by the speaker based on objective condition or subjective judgment.

The great similarity and slight differences between the two words in each confusable pair lead to enormous linguistic uncertainty and cognitive ambiguity, which will be illustrated by more examples in section 5 in terms of intralingual differences among Chinese modal verbs. Complexity caused by intricate form-meaning mapping between these polysemous modal verbs can result in psychological anxiety among the learners and cause errors in the use of modal forms. To learners from other countries, they may be unclear about the semantic nuances between Chinese modal verbs and uncertain about which is the right one to use in certain communicative contexts.
Based on frequency distribution of the different meanings of "neng", Wang (2000) established its semantic network and explained why the polysemous feature of "neng" created no difficulties for listeners who are native speakers of Chinese [7]. However, the perception of Chinese modals by learners of Chinese might be different from that by native speakers of Chinese. As Ely (2002) pointed out, "when native speakers receive linguistic input intended for them, they are relatively certain of its meaning" [4]. But learners may not be certain of it, as they have not developed an intuitive-like internal mechanism for modal comprehension as described in Wang's study in terms of native Chinese speakers. Due to the lack of such an internal mechanism for modal comprehension, with so many polysemous modal verbs to choose from and to tell apart, learners may be faced with great uncertainty as to what the modal verb means and which modal verb is the appropriate one to use. By identifying modal ambiguities from the semantic perspective, this paper is meant to help teachers make the learners understand that polysemy is an inherent feature of Chinese modal verbs, so the feeling of ambiguity about modal meaning and modal use is a natural phenomenon in acquisition, which has nothing to do with the learners' learning ability. Hopefully, this will help to reduce their psychological anxiety and raise their confidence in learning, consequently to increase their tolerance of ambiguity.

\subsection{Semantic Ambiguity Caused by the Indeterminate Feature of Modal Verbs}

The above analysis shows the polysemous feature of Chinese modal verbs, which does not pose problems for native speakers of Chinese, but may result in indeterminacy (uncertainty) felt by learners of Chinese from other countries when they perceive the meanings of Chinese modal verbs.

Both Coates (1983) [8] and Palmer (1990) [1] pointed out that indeterminacy is the basic feature of English modal verbs. It is the same case in terms of Chinese modals. Each Chinese modal verb has both root meanings and epistemic meanings. Sometimes it might be either this meaning or that meaning, sometimes it might be both this meaning and that meaning, or at different times it might be at different levels of a certain meaning. Such ambiguity or fuzziness leads to indeterminacy in understanding and using modal verbs. Coates (1983) held that the understanding of indeterminacy is the key to the understanding of modal verbs [8]. Because of this, she introduced the Fuzzy Set Theory which was originally used in mathematics into the field of semantic analysis to illustrate the fuzziness of modal verbs [8]. Fuzzy set figures were used by her to vividly reveal the indeterminacy of modal semantics. Through the fuzzy set figures, the either-or relationship, both-and relationship and gradient relationship of modal meanings can be clearly shown. In her book, she referred 
to the above three semantic relationships as ambiguity, merger and gradience [8]. But this paper regards all the three relationships as semantic ambiguities, because the term "ambiguity" in this paper is used in a broad sense, which means "uncertainty" or "lack of determinacy" [4]. According to Coates, all the three semantic relationships show indeterminacy of modal meanings [8]. Understanding ambiguity as indeterminacy/uncertainty, this paper refers to the above three types of semantic relationships as either-or ambiguity, merging ambiguity, and gradient ambiguity.

Both the root meanings and epistemic meanings of modal verbs have fuzziness, and they belong to different fuzzy sets, i.e. each meaning of the modal verb forms a fuzzy set. According to the fuzzy set figures drawn by Coates [8], there is a core in each fuzzy set, surrounded by skirt and periphery. The differences among core meaning, skirt meaning and periphery meaning of a modal verb represent the gradient ambiguity: different levels of a certain meaning, as illustrated in each fuzzy set shown in Figure 2. The darkened center is the core, the rough circle outside the core is the skirt, and the area outside the circle is the periphery.

Fuzzy set figures can be used to vividly represent the indeterminate meanings of English modal verbs [8]. This paper will apply fuzzy set figures to the semantic analysis of Chinese modals. For example, one of the root meanings of “能” (neng) is to express ability, and its epistemic meaning is to express possibility. In the Chinese sentence “她怎么能(neng)跑得那么快? (How could she run so fast?)", "neng" is equivalent to "can/could", which can mean root ability $\left(=\right.$ neng $_{1)}$ or epistemic possibility $(=$ neng ${ }_{4}$ ). These are two discreet meanings which belong to two fuzzy sets: fuzzy set of root meaning and fuzzy set of epistemic meaning, as shown in Figure 2. Depending on the context, "neng" in the above Chinese sentence shows either-or ambiguity, i.e., it either belongs to the root fuzzy set (indicating ability) or the epistemic fuzzy set (indicating possibility), as this Chinese sentence either asks how she managed to have the ability to run so fast (showing that the speaker feels curious) or questions how it was possible that she ran so fast (showing that the speaker feels incredulous). Either-or relationship in terms of modal meaning is one aspect of indeterminacy. In the absence of a specific context, it is hard to determine which meaning the modal verb refers to. It might be either one of its several discreet meanings (fuzzy sets), without merging area or overlapping area, as shown in Figure 2.

The either-or ambiguity may pose great difficulty for the learners, as they may not be certain about which meaning the modal verb really expresses, and a mistake in comprehension of the exact modal meaning may bring about breakdown in communication.

What is more complicated is that “能”(neng) has got more than two semantic meanings, with the 4 most frequently used meanings shown in Figure 3, including 3 root meanings and 1 epistemic meaning. But more often than not, its meaning in a particular context is hard to define as a discreet meaning, as it might be the combination of two meanings, located in the overlapping area of two fuzzy sets, resulting in merging ambiguity. For example, “陆明相信他的眼睛能(neng)治好, 小地方的医院治不 好他就考虑去大城市的医院治 (LU Ming believed that his eyes could be cured, he would consider going to hospitals in a bigger city)". In this Chinese sentence, the semantic meaning of "neng" is a combination of both root modality and epistemic modality, with a merging area, formed by neng 2 (expressing condition as a root meaning)

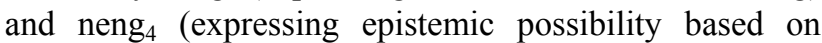
inference), meaning that he believed it was possible for his eyes to be cured under a certain condition (for example, in a bigger hospital). Merging ambiguity like this may lead to linguistic uncertainty in the comprehension of modal meaning, which may baffle learners and increase their anxiety.
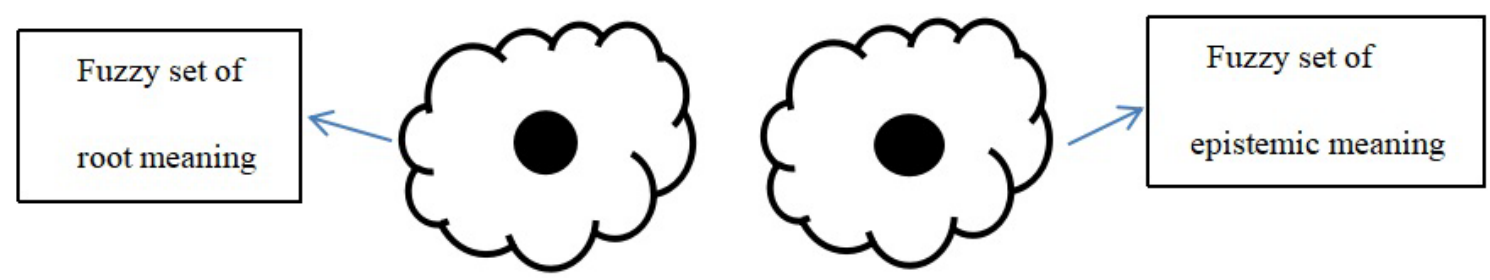

Figure 2. The gradient ambiguity and either-or ambiguity of modal verbs 




Figure 3. Multiple fuzzy sets of "neng": merging ambiguity

In the above figures, the core of the fuzzy set stands for the strongest sense of a certain meaning, but data from the study by Coates (1983) shows that the meaning of most modal verbs does not lie in the core area of a fuzzy set or in the area of a distinct fuzzy set [8]. It may lie in the skirt area, merging area and possibly either this area or that area, causing gradient ambiguity, merging ambiguity and either-or ambiguity.

It was Coates [8] who introduced the fuzzy set figures into the field of semantic analysis for linguistic research, but she didn't elaborate on its application in teaching. As the application of fuzzy set figures in semantic analysis can effectively show the indeterminate feature of modal verbs, it is proposed in this paper that they should also be applied in the teaching of modal verbs so as to help learners understand both the distinction between the multiple meanings of each modal verb and the fuzziness of modal meanings as an inherent feature of modal verbs in the hope that they can become more tolerant of ambiguity. Introducing fuzzy set figures into the teaching of Chinese modal verbs can vividly illustrate the different semantic meanings of a modal verb and may cognitively promote modal acquisition.

\section{Syntactic Ambiguity Caused by Inconsistencies in Root-epistemic Distinction}

The inconsistencies in grammatical rules for root-epistemic distinction can be revealed by looking at the double negative forms and interrogative forms of Chinese modal verbs.

The double negative form of Chinese modal verbs expresses affirmative meaning, which in English is often expressed by an affirmative modal verb. The double negative form of modal verb “能”(neng) — “不能不”(bu neng bu) - expresses root modality, not epistemic modality. It is usually used to show obligation, which is a root meaning, for example, “这样的事你不能不管 (You have to do something about it)". However, the double negative form of modal verb “会” (hui) — “不会不” (bu hui bu) - can be used to express both root modality and epistemic modality [5]. For example, in the Chinese sentence “他不会不知道, 是知道了不说 (He must have learned about this, he just kept silent about it)", “不会不” (bu hui bu) is used in an epistemic sense showing inference. This shows that the rule for root-epistemic distinction which applies to "neng" does not apply to "hui". Actually, there is not a consistent rule for root-epistemic distinction that applies to all modal verbs.

The interrogative form of “能”(neng) — “能不能”(neng bu neng) - can only be used to express root modality, for example, to show a request, as in “你能不能给我们做个 讲座? (Could you please give us a lecture?)". However, the interrogative form of “会”(hui) — “会不会”(hui bu hui) — can be used to express both root modality and epistemic modality [5], for example, to show epistemic inference, as in “他会不会已经走了? (Could he have gone?)”.

The above examples reveal that neither the double negative form nor the interrogative form of Chinese modal verbs is a marker of root-epistemic distinction for all the Chinese modal verbs. It applies to some modal verbs (such as "neng") as a marker of root-epistemic distinction but does not apply to other modal verbs (such as "hui") as such a marker. In other words, there are more than one rule for root-epistemic distinction for modal verbs, but none of these rules apply to all the modal verbs, because there are always some exceptions [5]. The existence of exceptions shows internal inconsistencies in the grammatical rules related to root-epistemic distinction.

Apart from rules for root-epistemic distinction, there are inconsistencies in terms of rules for other grammatical functions, too. For learners who are intolerant of ambiguity, they may perceive inconsistencies as a source of psychological discomfort or threat. The plethora of inconsistencies in grammatical rules for Chinese modal verbs may thus bring about syntactic ambiguity, which means ambiguous and complex situations in terms of modal grammar that may make learners feel uncertain. To reduce the feeling of uncertainty, it is advisable to identify all the inconsistencies in modal grammar and acquaint the learners with them in teaching. Their tolerance of ambiguity might be increased by understanding inconsistencies as an inherent feature of Chinese modal grammar.

\section{Mixed Ambiguity Caused by Intralingual and Interlingual Differences}

As was pointed out earlier, the potential for experiencing linguistic uncertainty is even greater when the learners reach a level where they begin to consider situational appropriateness and lexical nuance [4]. The syntactic requirements which derive from situational context, when 
combined with semantic nuances between the modal verbs, will cause a mixed type of ambiguity for learners of Chinese, who, being unaware of the syntactic contexts and the semantic differences, may make errors when understanding and using modal verbs. Mixed ambiguity can be analyzed from both intralingual and interlingual perspectives.

\subsection{Mixed Ambiguity Caused by Intralingual Differences}

In terms of second language learning, tolerance of ambiguity refers to the degree that learners tolerate a new linguistic system which is different from his/her mother tongue [3]. Differences between the two linguistic systems may cause ambiguity for the learners, for example, being uncertain about the different spelling systems, the different grammatical systems, etc. However, what causes ambiguity is not only the interlingual differences between the two linguistic systems but also the intralingual differences within the new linguistic system itself as a different system. Ambiguity in distinguishing different Chinese modal verbs causes learning anxiety. In terms of the modal system in Chinese, both the inconsistencies in syntactic rules and the complexity in semantic interpretations cause ambiguity. Inconsistencies in terms of syntactic rules have been exemplified in the previous section, this section will focus on the cognitive differences between modal verbs which have similar meanings but are used in different syntactic contexts.

For example, both “会”(hui) and “能”(neng) can express volition (this particular meaning of the two modal verbs is indicated respectively as hui $i_{1}$ and neng ${ }_{5}$, which forms a confusable pair of modals), however, their interrogative forms “会不会” (hui bu hui) and “能不能” (neng bu neng) are used in different contexts to ask about the listener's volition. The Chinese sentence “你会不会(hui bu hui) 听 他的?(Would you listen to him?)" asks about the volition (which is an internal force) of the listener, not in any way to benefit the speaker. But in the Chinese sentence “你能不 能(neng bu neng) 唱个歌我们听听? (Could you please sing a song for us?)", though it seems to ask about the volition (an internal force) of the listener whether he/she would like to sing a song, it is actually a request of the speaker for the listener to sing a song, so it is in a way for the listener to provide a service that benefits the speaker, who actually has imposed a kind of external force on the listener [5]. From these examples, the cognitive differentiation between Chinese modals which express similar meanings can be seen, and the possible confusion between them might cause ambiguity for the learners.

In the epistemic sense, “会”(hui) and “能”(neng) also have meanings which are cognitively quite similar, but there are semantic nuances between them and different syntactic situations for using them. For example, both "hui ${ }_{2}$ " and "neng," express epistemic prediction/inference, but according to the interpretation by semantic components, "hui ${ }_{2}$ " means subjective judgment of the speaker to show epistemic necessity (prediction of something that will necessarily happen), while "neng" means inference/judgment based on objective conditions to show epistemic possibility, not a necessity. When expressing epistemic meanings, "hui ${ }_{2}$ " can be used in affirmative, negative or interrogative sentences, while "neng ${ }_{4}$ " can only be used in negative and interrogative sentences [5]. This means neither the semantic boundary between them nor the syntactic conditions for using them are differentiated in a very clear-cut manner.

In one word, the intralingual differences between Chinese modal verbs are not clear-cut. It is not that in certain cases only "hui" is appropriate and in other cases only "neng" is appropriate. In some cases, both of them can be used, only when there are slight semantic differences and certain syntactic restrictions. Blurred distinction between them from the perspective of both semantics and syntax causes mixed ambiguity in understanding them and cognitive difficulty in using them.

\subsection{Mixed Ambiguity Caused by Interlingual Differences}

Since tolerance of ambiguity as a cognitive style means the degree that learners tolerate differences of a new system [3], the learning of a new language involves a cognitive process of adjustment and adaption to the new system. This is a process which requires great efforts because there is no "one-to-one correspondence" [4] between two linguistic systems. Cognitive differences between the two systems form an information gap which causes uncertainty and unpredictability. The following modal errors in the Chinese sentences made by English speakers reveal the wrong mapping between English and Chinese modals. The corresponding modal forms in the two languages (such as "can" in English, equivalent to "hui" or "neng" in Chinese according to the contexts) are partly similar and partly different both in meaning and in usage, easily causing confusion and leading to linguistic errors in communication.

Erroneous sentence 1: 只有勤奋的人才会(hui)取得成 功. “会”(hui) in this sentence should be replaced by “能”(neng) to mean possibility of something under a certain condition. The English version of the sentence is: only hard-working people can achieve success. In the Chinese sentence, “只有 (only)... 才 ...” introduces a condition and shows a syntactic context for "neng" to be used instead of "hui", because "neng" has "condition" as one of its meanings while "hui" has not [5]. In this sentence, English speakers may tend to map "can" in English with "hui" in Chinese, as there is indeed a semantic similarity between them, and they are equivalent to each other most of the time as both of them can mean root ability and epistemic possibility. But besides those two meanings, 
"can" in English can mean "condition" while "hui" in Chinese can not. Minor interlingual differences between "can" and "hui", together with stricter syntactic requirements in Chinese modal system cause learners from English-speaking countries to make transfer errors like the above when using Chinese modal verbs. This type of errors is brought about by the confusion between seemingly correspondent English and Chinese modals. From this example it can be seen that "can" in English can be mapped with not only "hui" but also "neng", depending on syntactic contexts.

Erroneous sentence 2: 王太太现在不能(bu neng)在家, 我刚刚看见她出门去上班了. In this negative sentence, “能” (neng) should be replaced by “会”(hui), which means an epistemic judgment by the speaker of this sentence, as the negative form of "hui" (which is "bu hui") can mean a negative judgment, while the negative form of "neng" (which is "bu neng") usually means "forbiddance" [5], which is a root meaning, not an epistemic judgment. The English version of the sentence is: Mrs. Wang can't be at home now, I just saw her leaving for work. "Can't" (the negative form of "can") in this English sentence expresses impossibility, i.e. a negative judgment. In other situations, "can't" can also mean "forbiddance", which in Chinese is correspondent to the negative form of "neng". This shows that "can" in English (and its negative form "can't") can be mapped with more than one modal form in Chinese (both "hui" and "neng", in positive or negative forms), expressing different meanings. Learners from English-speaking countries made the above error because they mapped the negative form of "can" in their mother tongue with the wrong negative form in Chinese, due to the confusion between negative forms of "hui" and "neng" as a confusable pair, which should be used in different syntactic contexts.

The above two erroneous sentences reveal not only intralingual differences between Chinese modals but also interlingual differences between English modals and Chinese ones: "Can" in English has a wider semantic coverage than either "hui" or "neng" in Chinese. "Can" in English can be used as both root "hui" and epistemic "hui", both root "neng" and epistemic "neng". It covers the most meanings of "hui" and all the five meanings of "neng". While both "hui" and "neng" can mean what "can" in English mostly means, they are used in different contexts and have more syntactic restrictions. Comparatively, the meaning range of "can" is much wider and is not restrained by linguistic and syntactic environments (such as negative sentences) [9]. Thus, the English modal form "can" might be mapped with two or more modal forms in Chinese ("hui" and "neng", etc.), depending on the syntactic context and semantic variation. This shows there are more syntactic restrictions on the use of Chinese modals. The meanings that could be expressed by the same English modal need to be expressed by different Chinese modals as the context varies, which results in not one-to-one correspondence but one-to-more mapping in terms of interlingual modal relationships. This kind of intricate mapping makes it especially difficult for learners of Chinese from other countries. To learners of low tolerance of ambiguity, Chinese modal system seems to cause a lot of confusion and ambiguity, thus they may feel inhibited in modal acquisition or even reject adjusting to the Chinese modal system which is so incongruent with their mother tongue system.

Because of the interlingual differences, interference from English makes it very likely for learners of Chinese to make transfer errors in the use of Chinese modals. Exploration of interlingual differences can help teachers to predict the difficulties in modal acquisition and the source of modal errors. Intralingual difference is another source for confusion and errors, as shown in the previous section. Due to both intralingual and interlingual differences, for learners of Chinese from other countries, the polysemous meanings of Chinese modal verbs are hard to take hold of, and Chinese modal verbs with similar meanings are hard to differentiate. Inadequate understanding of the syntactic restrictions for different Chinese modals and their meanings may easily cause ambiguity in their perception of modal meaning, and then lead to errors in form-meaning mapping between the two languages. Error analysis of interlanguage in terms of modal use by learners of Chinese can reveal what is difficult for the learners, and can enlighten the teachers in terms of error correction strategies. Teachers need to make learners understand there is no one-to-one correspondence between English and Chinese modal verbs. Thus, ambiguity perceived due to the above interlingual and intralingual differences might be better tolerated by learners.

\section{Conclusions: Modal Acquisition, Chinese Teaching, and Tolerance of Ambiguity}

As is shown above, due to intralingual complexity within Chinese modal system and interlingual differences between English and Chinese, learners of Chinese from other countries may be face with enormous cognitive difficulty when mapping the form and meaning of modal verbs. According to the hierarchy of difficulty in terms of second language acquisition [10], the one-to-more correspondence between form and meaning within the Chinese modal system and the one-to-more correspondence between modal verbs in the two languages (one modal form in the mother tongue is mapped with two or more modal forms in the target language) may be the most difficult for the learners to learn. "The level of difficulty experienced by the learner will be directly related to the degree of linguistic differences between the L1 and L2" [10]. When learning the Chinese modal verb, learners from other countries are faced with huge differences 
between Chinese and English modal systems. The appropriate use of modals depends on their understanding of both syntactic restrictions and semantic nuances within the Chinese modal system. Insufficient understanding of the above two will bring about ambiguity, which might be perceived by the learners as a threat and may lead to failure in modal acquisition.

"Tolerance of ambiguity merits consideration in teaching ESL" [4]. To identify and explore semantic, syntactic and mixed ambiguities that learners might be unsure about is to raise their awareness about linguistic uncertainty and increase their tolerance of ambiguity. As Ely (2002) suggested, the first step to help learners is to raise their consciousness about ambiguity and have them faced up to uncertainty [4]. In terms of modal acquisition, teachers can present learners with handouts which exemplify different types of modal ambiguity and acquaint them with the psychological conduct of tolerance of ambiguity, making them understand that it is natural for them to feel uncertain about the Chinese modal system and it is thus not necessary for them to have psychological fear about ambiguity. Thus, learners may view modal items characterized by uncertainty in a more positive way and no longer feel threatened by either interlingual or intralingual differences. To identify semantic, syntactic and mixed ambiguities when teaching Chinese modals is to reduce linguistic uncertainty and increase psychological tolerance. The ambiguities identified above can be used as a key to unlock more exploration of ambiguities in the Chinese modal system.

\section{Acknowledgement}

This paper is part of the research results from Humanities and Social Sciences Project (15YJA740017) which is funded by China Ministry of Education. I'm grateful for its financial support on the study of Chinese modal acquisition.

\section{REFERENCES}

[1] Palmer, F. R. Modality and the English Modals. London: Longman Group Limited;1990.

[2] Celce-Murcia, M., \& Larsen-Freeman, D. The Grammar Book: An ESL/EFL Teacher's Course ( $2^{\text {nd }}$ edition). Boston, MA: Heinle and Heinle; 1999.

[3] Brown, H.D. Principles of language learning and teaching ( $2^{\text {nd }}$ edition). Englewood Cliffs. NJ: Prentice-Hall; 1987: 89-90.

[4] Ely, C. M. Tolerance of Ambiguity and the Teaching of ESL. In: Reid J. M. (ed.) Learning Styles in the ESL/EFL Classroom. Beijing: Foreign Language Teaching and Research Press; 2002: 87-93.

[5] Lai, P. A Multiple-Perspective Study on English Speakers' Acquisition of Chinese Modal Verbs. Guangzhou: Sun Yat-sen University Press; 2016:46-62; 71-72;103-116; $167-168$

[6] Palmer, F. R. Mood and Modality ( $2^{\text {nd }}$ edition). Cambridge: Cambridge University Press; 2001: 7-10.

[7] Wang, W. Semantic Representation of Mandarin Modal neng (can) in Communications. Studies of the Chinese Language, 2000 (3): 238-246.

[8] Coates, J. The Semantics of the Modal Auxiliaries. London: Croom Helm Ltd.; 1983: 5-22.

[9] Lai, P. A Study on the Causes of Interlingual Transfer Errors in the Acquisition of Chinese Modal Auxiliaries. Language Teaching and Linguistic Research, 2006 (5): 67-74.

[10] Ellis, R. The Study of Second Language Acquisition. Oxford: Oxford University Press; 1994: 306-308. 